\title{
Efficient Annotation of Vesicle Dynamics in Video Microscopy
}

\author{
Leandro Cortés, Student Member, IEEE, and Yali Amit, Member, IEEE
}

\begin{abstract}
We describe an algorithm for the efficient annotation of events of interest in video microscopy. The specific application involves the detection and tracking of multiple possibly overlapping vesicles in total internal reflection fluorescent microscopy images. A statistical model for the dynamic image data of vesicle configurations allows us to properly weight various hypotheses online. The goal is to find the most likely trajectories given a sequence of images. The computational challenge is addressed by defining a sequence of coarse-to-fine tests, derived from the statistical model, to quickly eliminate most candidate positions at each time frame. The computational load of the tests is initially very low and gradually increases as the false positives become more difficult to eliminate. Only at the last step are state variables estimated from a complete time-dependent model. Processing time thus mainly depends on the number of vesicles in the image and not on image size.
\end{abstract}

Index Terms-Tracking, object detection, statistical object modeling, coarse-to-fine computation, multiple object configurations, fluorescence microscopy.

\section{INTRODUCTION}

$\mathrm{M}$ ODERN light microscopy can be coupled with digital recording to produce large data sets which must often be searched for specific organelles or sequences of events. We were faced with a problem of this type-the identification in movies of vesicles that fuse to the cell membrane [23]. Cells are genetically engineered to express fluorescent proteins on vesicles. During vesicle fusion, the fluorescent material is transferred from the vesicle membrane to the surface membrane and the material diffuses away from the point of fusion. Live cell videos are recorded by total internal reflection fluorescence (TIRF) microscopy [22]. The video of one cell may include tens or hundreds of vesicles per frame. Some are static, some move, and only a subset actually fuse. Of interest are submovies, i.e., the sequence of frames around the fusion time, constrained to a small window around the point of fusion. Fusion events often overlap in space and time. The signal-to-noise ratio is relatively low and the level of clutter produced by other fluorescent objects can be high. This is merely one example of a standard problem in many research fields in the biological and physical sciences. The scientist is interested in particular objects or events hidden in massive data sets of still or video images. Manual searching is slow and laborious, while ad hoc computerized search procedures are either inefficient or inaccurate.

- L. Cortés is with the Department of Computer Science, University of Chicago, 1100 E. 58th St., Chicago, IL 60637.

E-mail: leandro@cs.uchicago.edu.

- Y. Amit is with the Department of Statistics, University of Chicago, 5734 S. University Ave., Chicago, IL 60637. E-mail: amit@marx.uchicago.edu.

Manuscript received 21 Aug. 2007; revised 20 Jan. 2008; accepted 5 Mar. 2008; published online 1 Apr. 2008.

Recommended for acceptance by J.Z. Wang, D. Geman, J. Luo, and R.M. Gray.

For information on obtaining reprints of this article, please send e-mail to: tpami@computer.org, and reference IEEECS Log Number

TPAMSI-2007-08-0521.

Digital Object Identifier no. 10.1109/TSE.2008.84.
The main contributions of this paper are the use of a statistical model for an image presenting multiple objects and a coarse-to-fine algorithm for fast automatic annotation of the video according to different types of object dynamics. Each object is labeled by type of movement and a small number of state variables describing the movement. This then enables the scientist to retrieve not only the events of a particular label, for example, vesicle fusions, but, in addition, to actively filter the output in terms of the values of the state variable, thus selecting the most relevant submovies for the problem under investigation.

The advantage of statistical modeling lies in the ability to formulate well-defined hypothesis tests for the presence of objects and the flexibility to deal with multiple objects located in a small region. Statistical data models for individual objects, with clear rules to compose them online, provide models for object configurations and a principled method to assign weights, i.e., probabilities, to different possible interpretations of an image sequence. This together with the coarse-to-fine processing of motion data based on simplified object models are the novel contributions of this paper. Similar ideas of multiple object detection in the analysis of static images can be found in [2], [3].

The full gray-level statistical model for multiple moving vesicles is presented in Section 3, including a parameterization of the vesicle state, a data model given the presence of moving vesicles, and some prior assumptions on the possible vesicle states and motion. The steps of the detection procedure are then detailed in Section 4. Optimizing directly over the unobserved state variables for an unknown number of objects is computationally prohibitive. Instead, we use a coarse-to-fine computational approach for efficient detection and tracking.

The basic idea of the computation is to identify local functionals of the data that are invariant to certain state variables on one hand, but are still rare in generic background. In our case, these are binary-oriented edges 
which also exhibit some time discontinuity, as detailed in Section 4.2. We derive a simplified approximate statistical model in terms of these features. This is used to efficiently flag candidate locations and vesicle sizes. The candidates are then tested with a full statistical gray-level model for one or more vesicles, as described in Section 4.4. Candidates that survive this sequence of tests are tracked in time until they become undetectable, as described in Section 5 . Tracking involves measuring the location, width, and amplitude of a vesicle as it moves or fuses to the cell membrane, as well as deciding which of several motion categories is being observed. In Section 6.2, we describe some experiments on synthetic data to illustrate the error rates of the algorithm in the presence of different types of noise, both in terms of false positive and negative detections and in terms of the accuracy of the estimates of the vesicle state. In Section 6.3, we show some examples of the different scenarios in real data and present results on error rates in real data as well, based on manual labeling of the videos. We conclude with a discussion in Section 7.

\section{Related Work}

The problem of the detection and tracking of one or more objects can be treated as a two-step process that involves 1) detection, the identification of a target using the current data, and 2) tracking, the association of the detected target with an existing track or the generation of a new track. This separation between detection and tracking computation allows the use of a wide variety of object detection methods independently of the tracking method [10], [27], [1]. Tracking of multiple targets is usually approached using the Multiple Hypothesis Tracking (MHT) methods [6], [18] or Joint Probabilistic Data Association (JPDA) methods [17], [11]. MHT methods can handle several alternative data association hypothesis. Hypotheses are propagated into the future until new data solves uncertainty. JPDA methods estimate the joint distribution of the states of the targets based on the current and previous detections. The association among known tracks and new detections is done by estimating the probability $\beta_{i j}$ that the $i$ th detection belongs to the $j$ th track.

Methods that do not separate detection and tracking but, instead, do both computations simultaneously are referred in some literature as track-before-detect methods [26], [14]. These methods can show better performance when the quality of the data is affected by occlusion and clutter, which can considerably reduce the number of true positives generated by a detection algorithm. In this category, we can include several particle filter methods. These are Monte Carlo methods based on an approximation of the state distribution of the target using a sample. Most applications of particle filter methods deal with very few objects in a scene [12], [13]. Our method can be included in the trackbefore-detect category because part of detection and tracking computations are done at the same time. Moreover, when multiple objects are present in a small region, the tracking of the known targets and the confirmation or rejection of the candidate detections are done simultaneously in terms of the original unprocessed image data.
Most of the work in the field of detection and tracking of nano-objects using light microscopy has been focused on single fluorescent particles [8], [7], [21], [15], [16]. Particle detection methods also typically separate detection from tracking. They start by trying to enhance the quality of the data with image processing procedures such as matched filters, background subtraction, and image segmentation techniques [16]. Then, detection and tracking algorithms work on the processed data. In our method, image preprocessing is not viewed as a one-time computation that feeds information to the tracking algorithm with no further access to the original image data. All tests are derived from a statistical model for image data on and off object (foreground and background), thus avoiding arbitrary image processing techniques that can introduce artifacts in the data.

Single particle detection methods assume particles are isolated. They usually rely on simple semiautomatic image preprocessing techniques and thresholding for selection of candidate locations of particles. Subsequently, the location of each particle is estimated with subpixel resolution using methods based on intensity centroid, correlation, or least square fitting of an approximate point spread function (PSF). The initial efficient selection of candidate locations is an essential component of any computationally feasible algorithm. In our framework, such detections are based on approximations of the original statistical model defined in terms of binary-oriented edges, which makes this initial stage extremely fast (much faster than standard convolution or normalized convolution methods). Furthermore, a vesicle detected at a given frame immediately provides a candidate location and size for the subsequent frame.

After detection of candidate locations, several methods use a classifier to discard false candidate detections before fitting the approximate PSF model. Classification usually relies on the estimates of the moments on the intensities of a region around the candidate location [21], [25]. In our approach, such classification is defined in terms of hypothesis tests, which again are derived directly from the statistical data model or an approximation thereof, compared to an adaptive background model.

The image of a single particle can be approximated using the analytical model of the PSF of an aberration-free microscope or a simpler Gaussian-based model that approximates the analytical model. Gaussian models are preferred because they usually are computationally less intensive than the analytical model and produce better approximations of particle locations than non-PSF-based methods [20], [8]. For the case of vesicles, a Gaussian model has the additional advantage that it can be used to model vesicles of different sizes and it can approximate the images of vesicle fusions.

Single particle detection methods fail when partial overlapping occurs. Ad hoc measures of similarity or pretrained classifiers lack the flexibility to handle multiple objects. When the distance between particles gets smaller or below the diffraction limit, pixels may receive considerable intensity contribution from multiple particles, incurring a large error when single particle models are used to explain image data. Some work has been done on the detection of 
such particle configurations, showing improvements with respect to single particle methods [20], [16], [25], [24]. The problem of the detection of a particle configuration is set as fitting a linear combination of a number of (approximate) PSF functions, one per candidate particle. Differing numbers of particles correspond to different models, which are compared using tests based on mean square error ratios produced by pairs of models. Santos and Young [20] test a one-dot model against a two-dot model. Thomann et al. [25], [24] use a similar test, handling more than two particles with forward stepwise regression, comparing a sequence of models with increasing numbers of particles. To simplify computation at step $n+1$, they keep the estimated locations of the particles from step $n$, adding one additional particle at a nearby but unknown location. This is computationally intensive since multiple locations need to be tried. New random particles can be added to the model until the test rejects the augmented model. In contrast, we use a backward stepwise regression approach to select particles, initialized with 1) candidates from previous frames and 2) all new candidates that are found within a window by the efficient coarse testing procedure. We thus avoid creating phantom particles and the procedure is computationally more efficient. Using the information from $n$ candidates, we fit a linear combination of $n$ Gaussians to the data. The number of particles is defined by choosing only the $k$ Gaussians $(k \leq n)$ with coefficients that are statistically significant.

Once detection is finished on all image frames, tracking is used to connect detections from different frames in particle trajectories [21], [25], [24], [16]. In our method, detection and tracking are strongly connected. Indeed, a particle or particle configuration is tracked by updating all relevant state variables in a subsequent frame based on their values in the current frame. We assume that the appearance of a particle or particle configuration in one frame is similar to its appearance in the previous frame. Computing the simultaneous evolution of a particle configuration if more than one particle is present in a region significantly reduces the need for computing correspondences between detections in individual frames. Note that vesicle tracking must handle different vesicle sizes and changes in size due to fusion. Thus, the state space is larger for the vesicle case than for the particle case. The strong coupling between detection and tracking used by our method helps to reduce the time of convergence of the fitting algorithm by limiting the search to a sensible neighborhood of locations and sizes.

Theoretical analysis of the detection procedure is difficult because the primary source of error is not noise but clutter, i.e., structures in the image that have similarities to the desired objects. It is difficult to define convincing theoretical models for clutter. In the pure white noise regime, [5] offers interesting theoretical work on the limits of detectability in terms of object size and noise. Here, detection is simply determining if a relatively simple geometric object, of constant intensity, is present in the image or not. It is shown that, at the boundary of detectability, if properly organized in terms of multiresolution analysis, the computation is on the order of the number of pixels in the image. An interesting analysis in the point process case can be found in [4].

\section{Statistical Model}

Vesicles can appear at any frame, they can subsequently stay at the same location for any number of frames, they can move in the field of view, or they can fuse. The image intensities near a vesicle are modeled by a bell-shaped function of variable width, location, and amplitude, to which are added a background baseline intensity level, together with white noise. When several vesicles are present in a region, the intensities of their respective bell-shaped functions are added. The data model thus adapts to the number of hypothesized vesicles in a region. From the graylevel image model, we derive a sequence of approximate models that yield computationally efficient tests, allowing us to quickly remove most configurations or, alternatively, efficiently flag a small number of candidate configurations. The tests are applied in sequence, each of increasing computational complexity, but applied to a much smaller collection of candidate instances. Thresholds are set so that the probability of a false negative in any of the tests is very small. Vesicle fusion is modeled as motion in which the width and amplitude of the bell shape function change in time. In contrast, a simply moving vesicle is modeled as the change in time of the location variable.

\subsection{Single-Vesicle Data Model}

The image of a vesicle is modeled by a bell-shaped function of variable width, location, and amplitude, to which are added a constant background and noise. The intensity of a vesicle at location $x=\left(x_{1}, x_{2}\right)$ is modeled as $I(x)=G_{\omega}(x)+C+\zeta_{\sigma_{\text {noise }}}$, where $G_{\omega}(x)$ is a bell-shaped function with state $\omega, C$ is a baseline, and $\zeta_{\sigma_{\text {noise }}}$ is i.i.d noise that follows a normal distribution with mean zero and standard deviation $\sigma_{\text {noise }}$. We define

$$
G_{\omega}(x)=A e^{-\|x-\mu\|^{2} /\left(2 r^{2}\right)},
$$

where $\omega=(\mu, r, A)$ is the state vector composed of the location $\mu$, the width $r$, the maximum intensity $A$.

We assume that $C \geq 0$ is smoothly changing over the image so that, when a small region is considered, it is reasonable to assume $C$ is constant. Thus, for each vesicle $\omega$, we may have a different constant value $C$ for the immediate region around $\mu$.

Diffusion predicts that the increment of $r$ during fusion is proportional to time; thus, a vesicle fusion event is modeled by increasing $r$ and decreasing $A$ with time. On the other hand, a moving vesicle is modeled by varying the location $\mu$ but keeping $r$ and $A$ constant. Ideally, the total intensity (integral of $G_{\omega}$ ) should be constant when the vesicle fuses or when it moves. However, in real data acquisition, the intensity of the light emitted by each fluorophore may change due to phenomena such as bleaching (the fluorescent signal fades away permanently) or displacement of the fluorophore on a vesicle or cell membrane. The intensity of the emitted light by a fluorophore changes as it moves because the amplitude of 
the exciting light varies relative to the center of the region of interest of the microscope.

\subsection{Multiple-Vesicle Data Model}

Multiple vesicles might be present in the same region, producing a vesicle configuration. The model of the image intensity $I(x)$ is then given by

$$
I(x)=C(x)+\sum_{i} G_{\omega_{i}}(x)+\zeta_{\sigma_{\text {noise }}}
$$

where $i$ is an index over vesicles and $C(x)$ is the background intensity at $x$

If we assume that the intensity values of a vesicle are negligible at distance greater than $\delta r_{i}$ from $\mu_{i}$, we can define a simpler model for the image intensities $I(x)$ :

$$
I(x)=C+\sum_{i:\left|\mu_{i}-x\right| \leq \delta r_{i}} G_{\omega_{i}}(x)+\zeta_{\sigma_{\text {noise }}},
$$

where $C$ is assumed constant. When the image intensity $I(x)$ receives significant contribution from only one vesicle, the model (3) reduces to

$$
I(x)= \begin{cases}C+G_{\omega_{i^{*}}}(x)+\zeta_{\sigma_{\text {noise }}} & \text { if }\left\|\mu_{i^{*}}-x\right\| \leq \delta r_{i^{*}}, \\ C+\zeta_{\sigma_{\text {noise }}} & \text { otherwise, }\end{cases}
$$

where $\omega_{i *}$ is the only vesicle close to $x$.

\subsection{Dynamic Model}

The dynamic changes in vesicle appearance are modeled in terms of a time series of vesicle states (trajectories) $\Omega_{i}=\left(t_{0, i}, t_{f, i}, \omega_{i}^{(t)}: t \in\left[t_{0, i}, t_{f, i}\right], m_{i}\right)$, for vesicle $i$. Here, $t_{0}$ is the time of birth or first appearance of the vesicle, $t_{f}$ is the time of death, $\omega^{(t)}=\left(\mu^{(t)}, r^{(t)}, A^{(t)}\right), t \in\left[t_{0}, t_{f}\right]$ is the time course of the vesicle, and $m$ denotes the type of dynamics of the vesicle (e.g., fusion, translation, etc.)

A prior distribution is imposed on the evolution of the shape state $\omega$ given the type of dynamics which has a Markovian form, $P\left(\omega^{(t+1)} \mid \omega^{(t)}, m\right)$, and constrains the state at time $t+1$, given the state at time $t$ and the type of dynamics. At this point, we only implement a quadratic constraint on the variation of the vesicle center, i.e.,

$$
\log P\left(\mu^{(t+1)} \mid \mu^{(t)}\right)=\text { constant }-\lambda_{\mu}\left\|\mu^{(t+1)}-\mu^{(t)}\right\|^{2} .
$$

The constraint is the same for all motion types. More refined priors are possible to incorporate assumptions on the smooth variation of other state variables such as the amplitude, the width, or the baseline level.

Given a video sequence of $T$ images, detection consists of finding a set of $k$ admissible vesicle trajectories $\left(\Omega_{1}, \Omega_{2}, \ldots, \Omega_{k}\right)$, for an unknown integer $k \leq K$, which maximizes the posterior

$$
\arg \max P\left(\left(\Omega_{i}\right)_{i=1}^{k} \mid\left(I_{t}\right)_{t=1}^{T}\right),
$$

where, conditional on the vesicles $\Omega_{i}$, the data in each image is assumed independent and given by (2). That is,

$$
P\left(\left(I_{t}\right)_{t=1}^{T} \mid\left(\Omega_{i}\right)_{i=1}^{k}\right)=\prod_{t=1}^{T} P\left(I_{t} \mid\left(\Omega_{i}\right)_{i=1}^{k}\right) .
$$

A solution to the detection problem is a set of vesicles $\Omega_{i}$, $i=1, \ldots, k$, for some unknown number $k$. We assume a uniform prior on $k$ in some bounded domain. We also assume a uniform prior on the initial location $\mu^{\left(t_{0}\right)}$ and a uniform prior in a small range for the initial radius $r^{\left(t_{0}\right)}$. Contributions from different vesicles to the same pixel are managed through the data term, as explained above (2).

\section{Detection}

It is impossible to directly optimize (6) over all possible configurations of $k$ vesicles. Note, however, that, because a vesicle has a birth time $t_{0}$, the only way for a trajectory $\Omega$ to have high probability is for the image data around $\mu^{\left(t_{0}\right)}$ in frame $I_{t_{0}}$ to be consistent with the model (1) and for the image data in the region around $\mu^{\left(t_{0}\right)}$ in the preceding frame $I_{t_{0}-1}$ to be consistent with the background model that assumes a smoothly varying baseline. Otherwise, a more probable configuration can be found by moving $t_{0}$ either backward or forward. Thus, our initial goal is to detect candidate locations in all images that satisfy these two conditions, namely, detecting candidate births.

In other words, we assume that any vesicle trajectory can be flagged by a strong signal in a single frame at some point near its birth, with some evidence of discontinuity with respect to previous frames. Once a frame $t$ and state $\omega^{(t)}$ are detected, the remainder of the trajectory can be tracked forward and even backward, as will be explained below.

\subsection{Coarse-to-Fine Detection}

The detection of all near birth vesicles in all frames is a massive optimization problem as well since the number of vesicles in each frame is unknown and different configurations of nearby vesicles yield different likelihoods. We, therefore, define a sequence of simplified models that yield efficient tests which quickly select a reasonable number of candidate locations in each frame. First, we define a simplified binary-oriented edge model, including a temporal discontinuity component, which allows us to ignore the amplitude state variable $A$. This leads to a simple and efficient edge-based test that eliminates the vast majority of possible locations. Subsequently, the precise shape parameters of candidate vesicles are estimated using the full static data model (3), which is adapted to the number of candidate detections in a region. This step can also eliminate detections if the estimated amplitude $A$ for any of the candidates is below a threshold. All of the tests are standard statistical tests derived from the hypothesized data distribution using conservative thresholds to avoid losing true positives.

The sequence of detection steps described in this section assumes only one vesicle is present in a subregion of the image $I$. In this case, the data model in the subregion $I_{R}$ will be given by (4) and the simplified version of the detection problem will reduce to finding regions $R$, where

$$
\begin{aligned}
& \underset{\omega^{(t)}}{\arg \max } \log P\left(I_{t, R} \mid \omega^{(t)}\right)>\tau \text { and } \\
& \underset{\omega^{(t-1)}}{\arg \max } \log P\left(I_{t-1, R} \mid \omega^{(t-1)}\right)<\tau^{\prime},
\end{aligned}
$$

for some appropriately chosen thresholds $\tau, \tau^{\prime}$. 


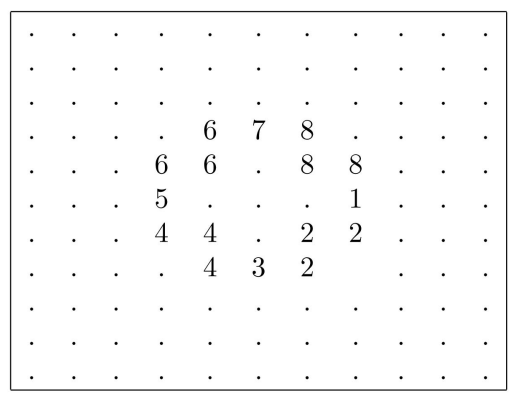

Fig. 1. Vesicle template. Example of a template for vesicles of radius 1.5 pixels computed from synthetic data. The numbers indicate the label $j$ for which $F_{j}\left(z_{i}\right)=1$; a dot means that $F_{j}\left(z_{i}\right)=0$ for all $j$.

\subsection{Edge-Based Models}

In the first step of detection, we aim at deriving a test that is invariant to amplitude and baseline variations in order to avoid the computationally intensive task of actually estimating these variables, for every candidate location. Detection, thus, starts using information only from locations with large variation in image intensity. We focus our attention on the spatial gradient of the vesicle template (2). For one vesicle $\omega=(\mu, r, A, C)$ with constant background, the maximum change in the gradient magnitude occurs on a circumference of radius $r$ centered at $\mu$.

We thus define a set of binary features defined in terms of the spatial gradient of the image, which is binned into $N_{F}$ disjoint ranges of gradient orientations. We define $F_{j}(x)=1$, if the magnitude of $\nabla I(x)$ is greater than a threshold $\phi$ and the gradient orientation is in the $j$ th range; otherwise, $F_{j}(x)=0$. Thus, at each pixel, we now have a vector of binary features $\hat{I}(x)=\left(F_{1}(x), \ldots, F_{N_{F}}(x)\right)$, for which at most one can be nonzero. It is important to observe that, by using only gradient orientation, we disregard information about the baseline $C$ or the amplitude $A$ of the vesicle. This is crucial from the computational point of view since it eliminates the need to apply normalized correlations to large templates everywhere in the image.

To incorporate the temporal condition related to the birth event, we eliminate those spatial features that do not correspond to a novel temporal event. This reduces the number of locations $x$ for which $F_{j}(x)=1$ due to static background structures, providing significant computational savings and reducing the number of false positives. We estimate the frequency of occurrence of the feature at pixel $x$ in the previous $M$ frames as $B_{F_{j}}(x)=\frac{1}{M} \sum_{t^{\prime}=t-M}^{t} \mathbf{1}_{F_{j}(x)=1}$. Assuming $F_{j}(x)=1$ at frame $I_{t}$, we accept the feature if $B_{F_{j}}(x)<\phi_{T}$. This can be viewed as a test of how likely the feature $F_{j}$ is under a background model, which is adaptively estimated using the previous $M$ frames.

The coarse model for a vesicle of width $r$ is a template containing $N$ features located at positions $z_{1}^{r}, z_{2}^{r}, \ldots, z_{N_{r}}^{r}$ on the circumference of radius $r$ and centered at the origin of a plane $Z$ (see Fig. 1).

Let $S_{j}$ be a set of indexes $i$ such that the feature at template position $z_{i}$ has label $F_{j}$. Formally,

$$
S_{j}=\left\{i: F_{j}\left(z_{i}\right)=1, i=1, \ldots, N\right\} .
$$

Since the amplitude and baseline variables have been eliminated, the instantiation for the coarse model is defined in terms of $\mu, r$. Given $\mu, r$, let $\theta_{i}(\mu, r)=\mu+z_{i}^{r}, i=1, \ldots, N_{r}$. We assume that

$$
P\left(F_{j}\left(\theta_{i}\right)=1 \mid \theta\right)= \begin{cases}p_{o} & \text { if } i \in S_{j}, \\ p_{b} & \text { otherwise }\end{cases}
$$

where $p_{o}$ is the probability of finding a feature $F_{j}$ at $\theta_{i}$, given that $F_{j}$ is the label assigned to the template position $z_{i}$. The probability $p_{b} \ll p_{o}$ is the probability of finding the feature $F_{j}$ anywhere else (off the instantiation or at $\theta_{i}$ with $i \notin S_{j}$ ). Assuming independence among all of the variables $F_{j}(x)$ given an instantiation $\theta(\mu, r)$, we write the likelihood of the transformed data conditional on $\theta$ as

$$
P(\hat{I}(x) \mid \theta)=\prod_{j=1}^{N_{F}}\left[\prod_{x \in S_{j}^{(\theta)}} P_{j}\left(x \mid p_{o}\right) \prod_{x \notin S_{j}^{(\theta)}} P_{j}\left(x \mid p_{b}\right)\right],
$$

where $S_{j}^{(\theta)}=\left\{\theta_{i}: i \in S_{j}\right\}$ and $P_{j}(x \mid p)=p^{F_{j}(x)}(1-p)^{1-F_{j}(x)}$.

In addition, we write the probability $P_{b g}$ of the data (assuming no vesicle in the image) as

$$
P_{b g}(\hat{I}(x))=\prod_{j=1}^{N_{F}} \prod_{x} p_{b}^{F_{j}(x)}\left(1-p_{b}\right)^{1-F_{j}(x)},
$$

which is a constant independent of the candidate instantiation value.

Dividing (9) by (10) and taking logs, we get

$$
\begin{aligned}
\log \frac{P(\mu, r \mid \hat{I}(x))}{P_{b g}(\hat{I}(x))}= & \log P(\mu, r)+n_{y}(\theta(\mu, r)) \rho_{1} \\
& -\left(N-n_{y}(\theta(\mu, r))\right) \rho_{2}+\text { const., }
\end{aligned}
$$

where $\rho_{1}=\log \left(\frac{p_{o}}{p_{b}}\right), \rho_{2}=\log \left(\frac{1-p_{b}}{1-p_{o}}\right)$, and $n_{y}(\theta)$ is the number of points $\theta_{i} \in \theta, i \in S_{j}$ that satisfy $F_{j}\left(\theta_{i}\right)=1$. The first test in the coarse-to-fine detection sequence simply consists of comparing $n_{y}(\theta(\mu, r))$ to a threshold $\tau_{H}$ which is set to minimize false positives.

Step 1: Hough transform. Instead of testing all of the possible centers $\mu_{\theta}$, we implement the Hough transform for the detection of circular objects. The transformation $\hat{I}(x)$ of the image $I$ produces a list $E$ of detected feature/location pairs $e=\left(F_{e}, x_{e}\right)$, where $F_{e}$ is the orientation bin of the gradient at the location $x_{e}$ in the image $I$. For fixed $r$, we detect subsets of $E$ that constitute admissible instantiations $\theta(\mu, r)$ for some $\mu$. The locations $\theta_{1}, \ldots, \theta_{N}$ of the features are points on a circle with center $\mu$ and radius $r$. The gradient at any point $\theta_{i}$ should have the same orientation as the normal to the circumference at the same position. Thus, if pair $e \in E$ belongs to a vesicle of radius $r$, the location of the center $\mu(e)$ would be

$$
\mu(e)=\left(x_{e, 1}+r \sin \left(F_{e}\right), x_{e, 2}+r \cos \left(F_{e}\right)\right) .
$$

The idea is then to have each detected pair $e \in E$ vote for the location $\mu(e)$ and then find those locations which have a vote count higher than $\tau_{H}$.

Since the data is never perfectly arranged on a circle and various deformations are possible, we have each feature/ location pair vote for a small set of potential centers as opposed to a single location. Let $I$ be an image of size $[m \times n]$ 


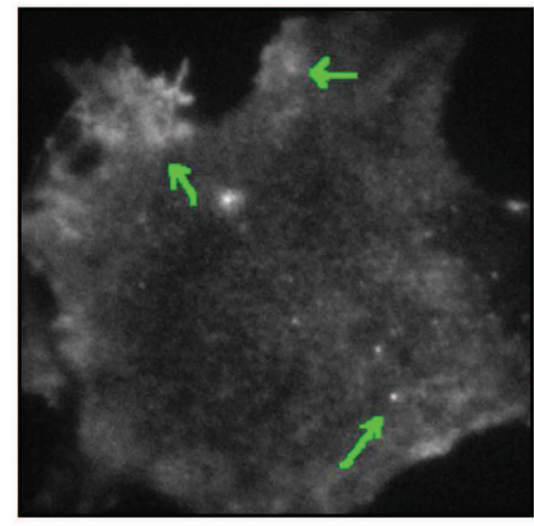

(a)

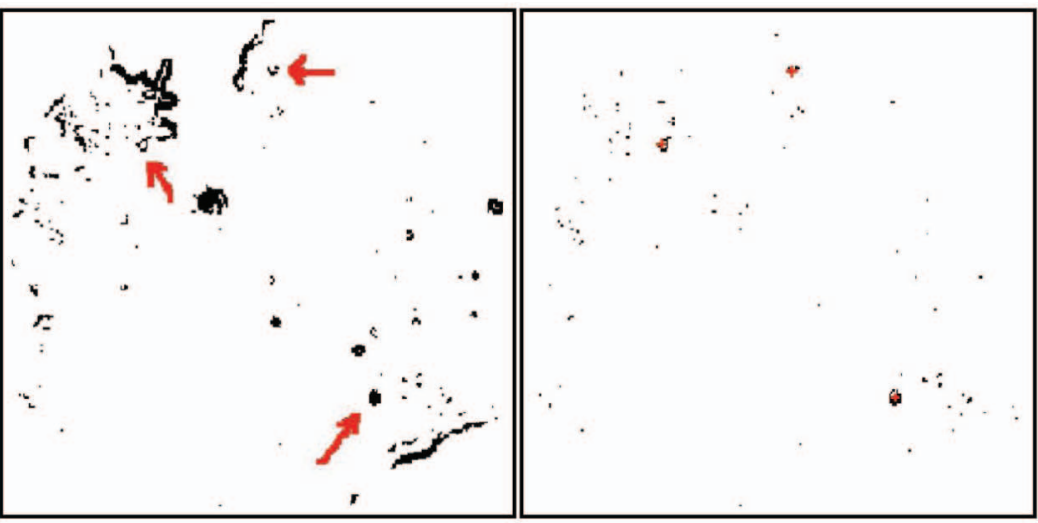

(b)

(c)

Fig. 2. Example of coarse detection. (a) Original frame. (b) Spatial features (thresholded gradient), arrows denote "new" vesicles. (c) Space-time features, the red " $+s$ " denote events detected with the Hough transform applied to these features. Observe the density difference between space features and space-time features. The Hough transform is used only to detect new objects; the bright vesicle in the upper left-hand quadrant of the image is not new and, hence, disappears with the space-time features.

and $B$ an array of size $\left[m^{\prime} \times n^{\prime}\right]$, where $m^{\prime}=m / b, n^{\prime}=n / b$, and $b \geq 1$. We map the locations $(i, j) \in I$ to locations $(\lfloor i / b\rfloor,\lfloor j / b\rfloor) \in B$. Each pair $e \in E$ now votes for the collection of circle centers represented by the bin in $B$ that contains the point $\mu(e)$. We choose as candidate centers $\mu$ only those bins from $B$ that received a vote greater than $\tau_{H}$. The value of $\tau_{H}$ is estimated from the data and used to control the number of false negatives. The above search through features simultaneously updates arrays $B_{r}$ corresponding to each possible radius size in some range.

The number of operations in this step depends only on the number of features detected in the image $|E| \ll m n$. Since these features are expected to satisfy both a spatial criterion and a temporal one, they are sparse, greatly reducing the size of $|E|$ (see Fig. 2).

Step 2: Improved circle detection. The features used by the Hough transform are computed using a very low threshold $\phi$ on the magnitude of the gradient, which produces wide bands of edge locations around discontinuities and, hence, a high number of false positives. Moreover, each selected pair $(b, r), b \in B_{r}$, corresponds to an entire set of candidate locations on the original image grid. To provide more precise estimates of $\mu$ and $r$, we first apply nonmaxima suppression on all spatial features obtained after computing the gradient of $I$.

Let $W_{b}$ be the finite set of locations corresponding to the selected bin and let $W_{r}$ be a finite set of reals around zero. We select $\mu$ and $r^{\prime}$ by finding the maximum of $n_{y}(\theta(\mu, r))$ over $\mu \in W_{b}, r \in W_{r}$. To account for small local deformations, we spread the features by 1 pixel, i.e., set $F_{j}(x)=1$ if a feature of type $j$ was found anywhere in the $3 \times 3$ neighborhood of $x$.

\subsection{Refined Models}

At the candidate locations that survived the previous tests, we compute the optimal values of the vesicle instantiation variables using the full gray-level model, which may involve several vesicles simultaneously. Assume first that there are no other detections in a region of interest $R$ of size $\delta r$ around a given detection $(\mu, r)$. We try to find the optimal value for the state variables in the image model defined in (4):

$$
\begin{aligned}
\underset{C, \omega \in \Omega}{\arg \max } L(\omega) & =\underset{C, \omega \in \Omega}{\arg \min } \sum_{x \in R}\left[I(x)-C-G_{\omega}(x)\right]^{2} \\
& =\underset{\mu, r, A, C}{\arg \min } \sum_{x \in R}\left[I(x)-C-A g_{\mu, r}(x)\right]^{2} .
\end{aligned}
$$

For fixed values of $\mu$ and $r$, we can estimate the global optimum of $A$ and $C$ using linear regression, where $g_{\mu, r}(x)$, $x \in R$, is considered the independent variable. This yields

$$
\begin{aligned}
& \hat{C}=\bar{I}-\hat{A} \bar{g}_{\mu, r}, \\
& \hat{A}=\frac{\sum_{x \in R}\left(g_{\mu, r}(x)-\bar{g}_{\mu, r}\right)(I(x)-\bar{I})}{\left(g_{\mu, r}(x)-\bar{g}_{\mu, r}\right)^{2}},
\end{aligned}
$$

where

$$
\bar{g}_{\mu, r}=1 /|R| \sum_{x \in R} g_{\mu, r}(x), \quad \bar{I}=1 /|R| \sum_{x \in R} I(x) .
$$

Let $R M S E=\sqrt{(R S S /|R|)}$ be the root mean square error with $R S S$ the residual sum of squares defined as

$$
R S S=\sum_{x \in R}\left(I(x)-\hat{A} g_{\mu, r}(x)-\hat{C}\right)^{2} .
$$

We optimize the likelihood in (13) by iterating between pairs of variables $(\mu, r)$, and $(A, C)$. For fixed $(\mu, r)$, the values $(A, C)$ are computed as in (14). Then, for fixed $A, C$, the cost is optimized in $(\mu, r)$ using conjugate gradient. This iteration is repeated until the relative change in the cost is small.

To decide whether a detection $\omega$ is significant, we test the hypothesis that $A$ is zero using the statistic $t_{A}=(\hat{A}-A) / \sigma_{\hat{A}}$, with

$$
\sigma_{\hat{A}}^{2}=\frac{s^{2}}{\sum_{x \in R}\left(g_{\mu, r}(x)-\bar{g}_{\mu, r}\right)^{2}},
$$

where $s^{2}=R S S /(|R|-2)$, computed at the final value of $(\mu, r)$.

The random variable $t_{A}$ follows a $t$-distribution with $|R|-$ 2 degrees of freedom [19] and we test at a significance level $\alpha_{\text {fine }}$ to decide whether a vesicle was detected. If the hypothesis that $A=0$ is not rejected, we reject the detection $\omega$. 


\subsection{Vesicle Configurations}

When the region $R$ includes $K>1$ vesicles, the model given in (3) should be used. In this case, we have to minimize

$$
J\left(\omega_{1}, \ldots, \omega_{K}, C\right)=\sum_{x \in R}\left[I(x)-C-\sum_{k=1}^{K} A_{k} g_{\left(\mu_{k}, r_{k}\right)}(x)\right]^{2},
$$

where $k$ runs over detections $\omega_{k}$ such that $\mu_{k} \in R$.

The methods used before apply in the multiple-vesicle framework. Now, for fixed $\mu_{k}, r_{k}, k=1, \ldots, K$, perform a multiple linear regression to solve for $C, A_{k}, k=1, \ldots, K$, and the conjugate gradient method is implemented simultaneously on all of the remaining variables.

When a candidate detection $\omega_{1}$ is at the center of the region $R$ and the candidate detections $\omega_{k}, k=2, \ldots, K-1$, are also inside $R$, we follow this procedure:

1. Repeat until none of the $A_{k}$ are rejected.

a. $\quad$ Try to fit the $K$ vesicles using a $K$-vesicle model.

i. Estimate all $A_{k}$ and $C$ with multiple regression.

ii. Estimate all $\mu_{k}$ and $r_{k}$ using the conjugate gradient algorithm.

b. Discard the fitted vesicles $\omega_{k}$ for which the estimated $A_{k}$ is rejected. The significance of each fit is determined using the statistic $t_{A_{k}}=\left(\hat{A}_{k}-\right.$ $\left.A_{k}\right) / \sigma_{\hat{A}_{k}}$ which also follows a $t$-distribution.

c. Update $K$.

2. Update the fitted values of $\omega_{k}, k=1, \ldots, K$.

\section{Tracking and Further Processing}

Tracking a (fusing or nonfusing) vesicle $\omega$ in time is performed for each detection surviving the previous stage. State variables of the vesicle are considered perturbations of the state variables in the previous frame, as expressed in (5). We therefore use the values $\omega^{(t)}$ estimated at frame $I_{t}$ as initial values in computing $\omega^{(t+1)}$ for the same vesicle in frame $I_{t+1}$. Thus, the tracking problem for a configuration of $K$ continuing vesicles in a region $R \subset I_{t+1}$ is to minimize

$$
\begin{gathered}
\frac{1}{\sigma_{\text {noise }}^{2}} \sum_{x \in R}\left[I(x)-C-\sum_{k=1}^{K} A_{k} g_{\left(\mu_{k}, r_{k}\right)}(x)\right]^{2} \\
+\sum_{k=1}^{K} \lambda_{\mu}\left\|\mu_{k}^{(t+1)}-\mu_{k}^{(t)}\right\|^{2} .
\end{gathered}
$$

The amplitudes $A$ and the baseline $C$ are recomputed using the regression model and the data in $I_{t+1}$, while $\mu_{k}$ and $r_{k}$ are kept fixed (same values from frame $I_{t}$ ). Then, a small number of iterations of the conjugate gradient algorithm are used to find the best values of $\mu$ and $r$ starting at initial values $\mu_{k}^{(t)}, r_{k}^{(t)}, k=1, \ldots, K$. We did not estimate the values of $\lambda_{\mu}$ systematically, rather, it was set based on some notion of the "fastest" motion we expect to detect, as explained in Section 6.

It is also possible for birth vesicles of frame $t+1$ to be located within a region $R$ containing continuing vesicles. In this case, the initial guess for $\mu_{k}^{(t+1)}, r_{k}^{(t+1)}$ for new vesicles
TABLE 1

Parameters Used to Process All Data Assuming the Pixel Intensities are in the Range [0, 1]

\begin{tabular}{|l|c|l|}
\hline$\alpha$ & 2.5 & parameter of the Deriche filter \\
\hline$\phi$ & 0.05 & threshold on gradient magnitude \\
\hline$N_{F}$ & 8 & number of edge orientations \\
\hline$M$ & 20 & length of temporal history \\
\hline$b \times b$ & $3 \times 3$ & size of a bin (Hough transform) \\
\hline$\tau_{H}$ & $0.4(2 \pi r)$ & vote threshold (Hough transform) \\
\hline$W_{r}$ & $\{1,2,3,4\}$ & coarse object sizes \\
\hline$\lambda_{\mu} \sigma_{\text {noise }}^{2}$ & .04 & relative weight of the dynamic model in (5) \\
\hline
\end{tabular}

will come from the coarse detection models, as described in Section 4.4 .

Tracking is done in the subsequent frames until a frame $t_{f}$ is reached for which no signal is detected, i.e., the hypothesis $H_{0}: A_{k}=0, k=1, \ldots, K$, is accepted.

\subsection{Fusing versus Nonfusing Vesicles}

The final products of the detection process are sequences $\omega_{t_{0}}, \ldots, \omega_{t_{n}}$, where $\omega_{t}$ is a vector with the fitted values of a detection at frame $t$. Each sequence represents the life of a single vesicle. To decide whether the vesicle did fuse or not, we use the series of fitted radii $r_{t}$. We assume that if a vesicle does not fuse, the values of $r_{t}$ should be affected only by the noise in the image. Recall that a fusing vesicle is modeled by increasing $r$ and reducing $A$. To decide if a vesicle has fused, we use the linear regression model $r_{t}=\beta_{1} t+\beta_{0}$. The standard $t$-test at some significance level $\alpha$ determines whether the slope $\beta_{1}$ is zero, in which case we conclude that $r$ did not change during the life of the vesicle and, therefore, did not fuse.

\subsection{Moving versus Static Vesicles}

To distinguish between moving and static vesicles, we use the series of estimated locations $\mu_{t}=\left(\mu_{1, t}, \mu_{2, t}\right)$ and fit a linear model to each coordinate $j=1,2$ :

$$
\mu_{j, t}=\beta_{j, 1} t+\beta_{j, 0}
$$

with $t$ varying from $t_{0}$ to $t_{n}$. Again, we conclude that there was a change in the object location $\mu$ if $\beta_{j, 1}$ is found to be significant at some level $\alpha$ for $j=1$ or $j=2$.

\section{EXPERIMENTS}

The detection and tracking procedure described above has been used on synthetic and real-world data. The synthetic images were generated using the data model with a variety of noise conditions. These synthetic experiments were aimed at measuring the performance of the algorithm in a controlled environment. The key parameters used in all experiments are listed in Table 1. In general, parameters are set to conservative values so that we can avoid the need to reset values for new videos. The set of circle sizes (coarse object sizes) is $\{1,2,3,4\}$. A radius of 1 pixel is just below the physical limits of light microscopy. Radii greater than 3 pixels are rare. These sizes depend directly on the numerical aperture of the microscope and the resolution of the CCD camera used for registration. Vesicle locations are assumed to be uniformly distributed over the image. For the case of tracking, we assume that 
TABLE 2

White Noise Detection Accuracy

\begin{tabular}{|c|cccc|}
\hline SNR & $r=1.0$ & $r=1.5$ & $r=2.0$ & $r=2.5$ \\
\hline \hline & & \multicolumn{4}{|c|}{ RMSE $(\mu)$} \\
4 & $0.353 \pm 0.271$ & $0.335 \pm 0.237$ & $0.357 \pm 0.237$ & $0.339 \pm 0.201$ \\
16 & $0.063 \pm 0.055$ & $0.048 \pm 0.039$ & $0.068 \pm 0.058$ & $0.072 \pm 0.060$ \\
64 & $0.007 \pm 0.006$ & $0.007 \pm 0.006$ & $0.007 \pm 0.006$ & $0.007 \pm 0.006$ \\
\hline \hline & \multicolumn{4}{|c}{ RMSE $(A)$} \\
4 & $0.238 \pm 0.178$ & $0.195 \pm 0.127$ & $0.183 \pm 0.095$ & $0.154 \pm 0.068$ \\
16 & $0.134 \pm 0.048$ & $0.124 \pm 0.022$ & $0.124 \pm 0.019$ & $0.124 \pm 0.015$ \\
64 & $0.122 \pm 0.007$ & $0.122 \pm 0.005$ & $0.122 \pm 0.004$ & $0.122 \pm 0.003$ \\
\hline \hline & & \multicolumn{4}{c}{ RMSE $(r)$} \\
4 & $0.317 \pm 0.296$ & $0.291 \pm 0.283$ & $0.269 \pm 0.254$ & $0.233 \pm 0.232$ \\
16 & $0.079 \pm 0.079$ & $0.020 \pm 0.020$ & $0.042 \pm 0.042$ & $0.047 \pm 0.046$ \\
64 & $0.004 \pm 0.004$ & $0.004 \pm 0.004$ & $0.004 \pm 0.004$ & $0.004 \pm 0.004$ \\
\hline
\end{tabular}

RMSE for the estimated values of $\mu, A$, and $r$ using synthetic data with different levels of white noise. Synthetic vesicles of size $r=1.0,1.5$, 2.0, and 2.5 were used.

$P(u(t+1) \mid u(t)) \sim \operatorname{Normal}\left(u(t), 4^{2}\right)$ in pixel units. The choice of variance is thus made large enough to account for fast moving vesicles. Taking $\sigma_{\text {noise }}=0.75$ which is quite large on a pixel range of $[0,1]$, the relative weight of the prior term is $\sigma_{\text {noise }}^{2} / 16=0.04$. No prior on amplitude $A$ or baseline $C$ is explicitly set, but negative values are rejected.

\subsection{Computation}

The experiments have been done on a computer with a Pentium 4 CPU running at $2.80 \mathrm{GHz}$ and 1.5 Gbytes of RAM. Data was read from a regular EIDE hard drive (WD1200JB-00DUA3 of 120 Gbytes, 100 Mbytes/s, 8 Mbytes Cache, 7,200 rpm). The program was written in $\mathrm{C}++$ and currently runs on a Linux machine (Mandriva 2006). The coarse detection steps: feature detection and Hough transform take $0.025 \pm 0.005 \mathrm{~s} /$ frame. The refined detection step (Gaussian fitting) takes $0.003 \pm 0.0014 \mathrm{~s} /$ vesicle. Thus, if 100 candidates per frame are produced by the coarse models, the refined detection process takes approximately $0.3 \mathrm{~s} /$ frame. Given the conservative threshold used by the Hough transform, there are very few false negatives at that step. Assuming (in a highly cluttered scene) several hundred detections of the Hough transform per frame, the computational gain would be on the order of 1,000 times, relative to fitting the full Gaussian model at each of the $256^{2}$ locations for each of the four radii.

The program works directly on raw digital videos. Gradient estimation is made using the filter developed by Deriche [9]. To satisfy memory constraints, the videos are processed in chunks. Detection and tracking are performed on each chunk using the procedure describe above. Then, backward tracking of vesicles is made to improve the tracking of vesicles when they approach the surface membrane. Once the video processing ends, vesicles are labeled according the type of movement they show. Thus, vesicles are labeled as fusing vesicles, static nonfusing vesicles, and moving vesicles.

\subsection{Synthetic Data}

Synthetic videos were generated using model (2). We repeated the experiments using data generated using the modified (but more realistic) assumption that noise is not additive but is Poisson distributed, with mean given by
TABLE 3

White Noise Error Rates

\begin{tabular}{|c|ccccc|c|}
\hline & \multicolumn{5}{|c|}{ False negatives $(\mathrm{N}=1421)$} & False Positives/frame \\
SNR & $r=$ & 1.0 & 1.5 & 2.0 & 2.5 & (1200 frames) \\
\hline \hline 4 & & 186 & 57 & 19 & 12 & 8.73 \\
16 & & 23 & 2 & 1 & 0 & 3.31 \\
64 & & 0 & 0 & 0 & 0 & 0.00 \\
\hline
\end{tabular}

Number of false positive and false negative detections under different levels of SNR for synthetic data + white noise. The number of true vesicles is 1,421 per each size. The number of false positives was measured over 1,200 image frames.

the image intensity $\lambda=I(x)$, which implies that the standard deviation is $\sqrt{I(x)}$.

\subsubsection{White Noise}

The synthetic images with white noise were generated with a constant baseline $C(x)$. A noiseless video $\left(\sigma_{\text {noise }}=0\right)$ with random synthetic vesicles was used as initial data. The experiments were repeated using the same base video after adding noise at different levels of signal-noise-ratio. Here, we define signal-noise-ratio as $S N R=A / \sigma_{\text {noise }}$.

The first experiment tested the accuracy of the algorithm at detection of vesicles as a function of $S N R$. Synthetic vesicles were located at random positions and had a lifetime of one frame. The radius $r$ of each vesicle was chosen from the set $\{1.0,1.5,2.0,2.5\}$. No spatial overlapping was allowed.

Table 2 shows the root-mean-square error (RMSE) and the standard deviation of the squared error for estimated values of $\mu, A$, and $r$ under several levels of $S N R$. The algorithm shows stable behavior for high levels of $S N R$, with higher error for the smallest vesicles $(r=1.0)$. The RMSE in location $\mu$ and radius $r$ is much smaller than 1 pixel, even for the lowest signal-noise ratio $(S N R=4)$.

Table 3 shows counts of false positive and negative detections produced by the algorithm. The number of false negative detections is correlated with the size of the vesicles and the SNR. High levels of noise affect the ability of the algorithm to detect small vesicles and small vesicles are less likely to be detected than bigger vesicles at the same SNR levels. The algorithm had the highest false negative rate when $r=1.0$ and $S N R=4$. For this case, the algorithm failed to detect 186 out of 1,421 , i.e., $\sim 13$ percent of the vesicles.

Table 3 also shows that a high number of false positive detections were produced for low signal-noise ratios. The coarse detection steps produce a high number of false candidate detections that survive the selection criteria. We conjecture that this high number of false detections for low $S N R$ conditions might be reduced by using an adaptive $p_{b}$ (probability of finding features in the background), estimated from earlier frames. A different value of $p_{b}$ implies different thresholds for the Hough transform and for the improved circle detection method (see discussion in Section 7).

The second experiment was aimed at testing the accuracy of the tracking algorithm. Synthetic fusing vesicles were located at random positions and had a fusion lifetime of 10 or 50 frames. The fusion was simulated by keeping the location $\mu$ constant and varying 
TABLE 4

White Noise Tracking Errors

\begin{tabular}{|c|cccc|}
\hline SNR & $r=1.0$ & $r=1.5$ & $r=2.0$ & $r=2.5$ \\
\hline \hline \multicolumn{5}{|c|}{ RMSE $(\mu)$ (vesicle lifetime $=10$ frames) } \\
4 & $0.568 \pm 0.449$ & $0.674 \pm 0.353$ & $0.605 \pm 0.223$ & $0.506 \pm 0.154$ \\
16 & $0.300 \pm 0.317$ & $0.153 \pm 0.096$ & $0.097 \pm 0.056$ & $0.082 \pm 0.054$ \\
64 & $0.043 \pm 0.022$ & $0.024 \pm 0.034$ & $0.016 \pm 0.031$ & $0.016 \pm 0.029$ \\
\hline \hline \multicolumn{5}{|c}{ RMSE $(\mu)$ (vesicle lifetime $=50$ frames) } \\
4 & $1.014 \pm 0.671$ & $0.902 \pm 0.573$ & $0.673 \pm 0.282$ & $0.559 \pm 0.190$ \\
16 & $0.327 \pm 0.182$ & $0.267 \pm 0.156$ & $0.228 \pm 0.148$ & $0.201 \pm 0.130$ \\
64 & $0.078 \pm 0.073$ & $0.047 \pm 0.053$ & $0.034 \pm 0.058$ & $0.027 \pm 0.055$ \\
\hline
\end{tabular}

RMSE when tracking location $(\mu)$ on synthetic data with different levels of white noise. Synthetic vesicles of size $r=1.0,1.5,2.0$, and 2.5 with lifetimes of 10 and 50 frames were used.

$r_{t}$ from $r_{0} \in\{1.0,1.5,2.0,2.5\}$ to $r_{t}=5.0$ while $A_{t}$ was constrained by $A_{0} r_{0}=A_{t} r_{t}$. No spatial overlapping among vesicles was allowed.

We computed the RMSE in the location $\mu$ for fusing vesicles with life of 10 and 50 frames. The RMSE was averaged over the frames that the vesicle was tracked. Table 4 shows the average RMSE as a function of SNR. We use the same definition of SNR from the first experiment, but we measure it at the initial frame only. Since the actual SNR decreases as the vesicle fuses, the computed average RMSE is higher than the RMSE computed in the first experiment.

\subsubsection{Shot Noise}

These experiments were repeated in a modified framework that assumes Poisson noise with intensity $\lambda(x)=I(x)$. For the experiments in this section, we show error as a function of the vesicle amplitude: $A \in\{0.16,0.32,0.64\}$ and sizes $r \in\{1.0,1.5,2.0,2.5\}$. Table 5 shows the error of the algorithm estimating $\mu, r$, and $A$. The RMSE in location increases as the intensity (amplitude) of the vesicle decreases. Higher $\operatorname{RMSE}(\mu)$ is produced for vesicles with larger size $r$. This behavior can be explained as follows: For a fixed amplitude, the mean intensity of the pixels covered by a vesicle is proportional to $r$. Since shot noise is proportional to intensity, the mean level of noise is higher for wider vesicles with the same amplitude $A$. The behavior of relative RMSE in amplitude $A$ is different from the RMSE in location. RMSE for the amplitude $A$ increases as $A$ and $r$ decrease. For lower values of $A$, the effect of noise in the background seems to be higher on the estimate of $A$.
TABLE 5

Shot Noise Detection Accuracy

\begin{tabular}{|c|cccc|}
\hline $\mathrm{A}$ & $r=1.0$ & $r=1.5$ & $r=2.0$ & $r=2.5$ \\
\hline \hline & & \multicolumn{4}{|c|}{ RMSE $(\mu)$} \\
0.16 & $0.031 \pm 0.026$ & $0.029 \pm 0.024$ & $0.040 \pm 0.035$ & $0.136 \pm 0.127$ \\
0.32 & $0.019 \pm 0.017$ & $0.019 \pm 0.016$ & $0.018 \pm 0.016$ & $0.027 \pm 0.024$ \\
0.64 & $0.010 \pm 0.009$ & $0.009 \pm 0.008$ & $0.009 \pm 0.007$ & $0.009 \pm 0.008$ \\
\hline \hline & \multicolumn{4}{|c}{ RMSE $(A)$} \\
0.16 & $0.020 \pm 0.005$ & $0.020 \pm 0.004$ & $0.020 \pm 0.003$ & $0.020 \pm 0.002$ \\
0.32 & $0.040 \pm 0.006$ & $0.039 \pm 0.004$ & $0.039 \pm 0.003$ & $0.039 \pm 0.003$ \\
0.64 & $0.078 \pm 0.008$ & $0.078 \pm 0.005$ & $0.078 \pm 0.004$ & $0.078 \pm 0.003$ \\
\hline \hline & & \multicolumn{4}{c|}{ RMSE $(r)$} \\
0.16 & $0.016 \pm 0.016$ & $0.016 \pm 0.016$ & $0.035 \pm 0.035$ & $0.034 \pm 0.034$ \\
0.32 & $0.009 \pm 0.009$ & $0.009 \pm 0.009$ & $0.009 \pm 0.009$ & $0.009 \pm 0.009$ \\
0.64 & $0.005 \pm 0.005$ & $0.005 \pm 0.005$ & $0.005 \pm 0.005$ & $0.005 \pm 0.005$ \\
\hline
\end{tabular}

RMSE for the estimated values of $\mu, A$, and $r$ using synthetic data with different levels of shot noise. Synthetic vesicles of size $r=1.0,1.5,2.0$, and 2.5 were used.

Under the shot noise model, the number of false positives and negatives was zero in almost all experiments. The only exception was for vesicles $(A=0.16, r=2.5)$. For this case, the algorithm missed 15 out of 1,421 vesicles. The values $(A=0.16, r=2.5)$ correspond to the hardest case in the experiment (lowest amplitude $A$ and largest $r$ ). Dim vesicles are less likely to pass the signal test. In addition, as explained for the RMSE plots, the error in location is higher for wider vesicles and the error in size $(r)$ is higher for vesicles with low amplitude $A$ relative to background $C$.

\subsection{Experiments with Real Data}

The real data was obtained using (TIRF) microscopy [22]. The videos show vesicle activity in the cell membrane, recorded at a rate of 33 frames/s. Each frame is a gray-level image of $256 \times 256$ pixels. Each pixel can take an integer value between 0 and 4,095. The duration of a movie can vary between 2 and $20 \mathrm{~min}$.

Once vesicles are detected, tracked, and labeled, submovies of isolated fusing vesicles are generated. Each submovie contains only the data from the region where the vesicle was detected. Several thousand of these small movies have been produced in our lab and they are used for statistical analysis of the kinetics of vesicle fusion. An example of a detected fusion in a cell is shown in Figs. 3 and 4.

\subsection{Examples of Different Detection Types}

An example of multiple-vesicle detection is illustrated in Fig. 5. A zoomed view of a region of a cell membrane is shown
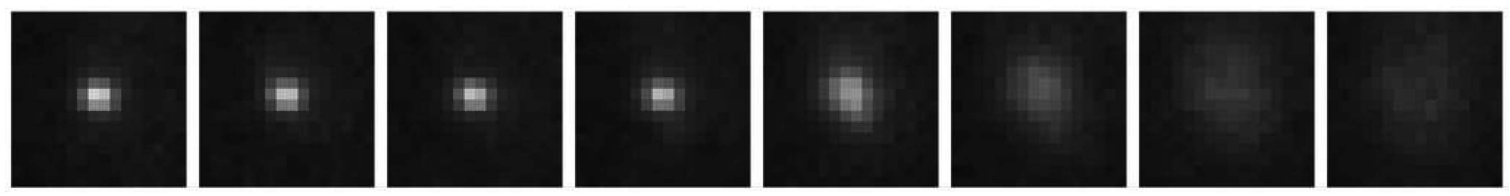

Fig. 3. Fusing vesicle. A sequence of frames of a region containing a fusing vesicle.
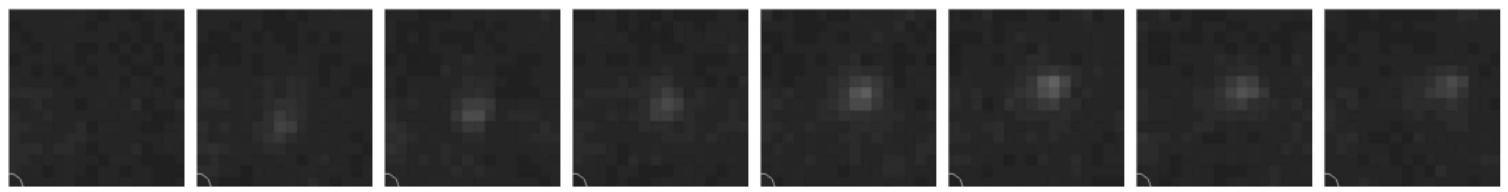

Fig. 4. Moving vesicle. A sequence of frames of a region containing a moving vesicle. 


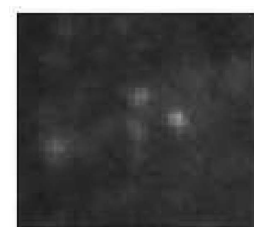

(a)

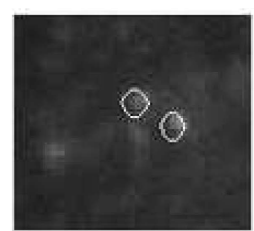

(b)

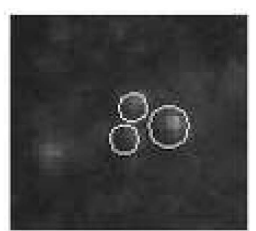

(c)
Fig. 5. Vesicle detection example. (a) A region with four vesicles. (b) Detections obtained using a single-vesicle model. (c) Detections obtained using a multiple-vesicle model.

in Fig. 5a. There are four distinguishable vesicles in the image: a cluster of three vesicles and one isolated vesicle.

The program produces three candidate vesicles located in the cluster. Using the single-Gaussian model, it is necessary to do the Gaussian fitting process three times, once per candidate detection. Each fitting is done on a different region centered at the candidate location. After testing for signal, one of the candidates is rejected. Fig. $5 \mathrm{~b}$ shows a circle for each detected vesicle using a singleGaussian model. The detection improves when the multiple Gaussian model is used. The fitting is done on one region covering the three circles in the cluster. The 3-Gaussian model passes the signal test. No candidate is rejected.

Finally, in Fig. 6, we show some examples of interesting configurations detected by the algorithm. Vesicles showing different types of dynamics are detected in the same region of a cell membrane during a short period of time ( $\sim 17$ seconds). Only 10 frames with new interesting events are presented. Estimated locations and sizes are depicted with circles. Color is used to indicate type of dynamics. An example of a static vesicle (purple) shows up in frame 2 and stays for the rest of the sample sequence. A new vesicle (yellow) appears in frame 4 and fuses to the cell membrane. The fusion event produces an apparent overlapping of two vesicles (frames 6-8). In frame 3, a short-lived vesicle (cyan) appears. In this case, the time series of values of $\mu$ and $r$ is too short for the algorithm to determine the type of dynamics of the vesicle. A false moving vesicle (red) is also shown in the example. The decision that the vesicle is moving is based on a time interval that is much longer than the sequence shown in this example.

A video illustrating some more detection types and their tracking can be found at www.stat.uchicago.edu/ amit/ track/video.

\subsection{Error Rates on Real Data}

Different drugs and engineered vesicle labels are used to study the kinetics of exocytosis. This experimental variability is reflected in the number of vesicles, the dynamic properties of the vesicles and the number of extraneous objects in the background. We present ROC curves of the performance of the algorithm on two very different cell experiments. For simplicity, we called the cells used in the experiments cell $A$ and cell $B$. The fluorescent labels of cell $A$ were engineered to fluoresce only during vesicle fusion. Accordingly, most of the vesicles observed in the video of this cell were fusing vesicles. However, a few static and moving vesicles were also present in the video. Cell B was similar, but it was treated with a drug that made the vesicle labels fluoresce before fusion as well. A huge increment in the number of fluorescent background objects and noise is therefore observed in this movie. Moving vesicles outnumber the static and fusing vesicles (see Table 6).

To generate ground truth, we performed a visual inspection and classification of all detections produced by the program using a very low threshold, essentially guaranteeing no false negatives. First, it was decided whether the detection matched a real vesicle. Then, for each good detection, a label was assigned indicating one of the following categories:

1. the vesicle is fusing,

2. the vesicle is moving,

3. the vesicle is static, and

4. undetermined behavior.

The uncertainty is due to two factors: the short duration of these vesicles in the video and the high levels of noise and clutter. Table 6 provides the numbers of each category for both cell types. Fig. 7 shows the different ROC curves obtained after processing the videos of cells $A$ and $B$. We plot the fraction of true positives detected against the number of false positives per frame. The program was run using the same parameters in both cases (Table 1). Detections in class 4 were discarded.

All vesicles. Fig. 7a shows the ROC curves for the detection of all vesicle types together. To define a classifier, we used a threshold on the signal level $t_{A}=\hat{A} / \sigma_{\hat{A}}$ measured at the frame where $A$ was maximum (see Section 4.3). In this case, false detections were those not considered to be real vesicles by the human classifier. False detections in cell $A$ and cell $B$ were often found on the cell boundary, where moving parts of the cell or noisy fluorescent bumps were mistakenly detected as vesicles. False detections in cell $B$ also included parts of fluorescent background objects (moving and static) that were detected as vesicles.

It is of interest that the mean and maximum signal levels found in cell $A$ were, respectively, $\sim 1.47$ and $\sim 1.77$ times larger than the mean and maximum signal levels obtained from cell $B$. This difference suggests a difference in the SNR of the videos, which may also account for more false detections in cell $B$.

Fusing vesicles. Fig. $7 \mathrm{~b}$ shows the ROC curves for vesicle fusions. The automatic classification was defined by a threshold on the $t$-value used for the test on the slope $\beta_{1}$, as defined in the linear model of Section 5.1. True positives were the detections that were assigned to category 1 by the human classifier. False vesicle fusions were produced mainly by interaction between static objects (bumps or static vesicles) with low signal and fluorescent molecules moving away from a nearby fusion. More static objects, i.e., clutter, and lower signal led to an increased rate of false detections in cell $B$.

Moving vesicles. Fig. 7c shows the ROC curves for moving vesicles. The automatic classifier was defined using the model from Section 5.2. True positives were the detections that were classified in category 2 by the human classifier. False detections were due mainly to the inherently low signal of moving vesicles and late fusing vesicles. The low signal of moving vesicles is due to their location in depth. Unlike 


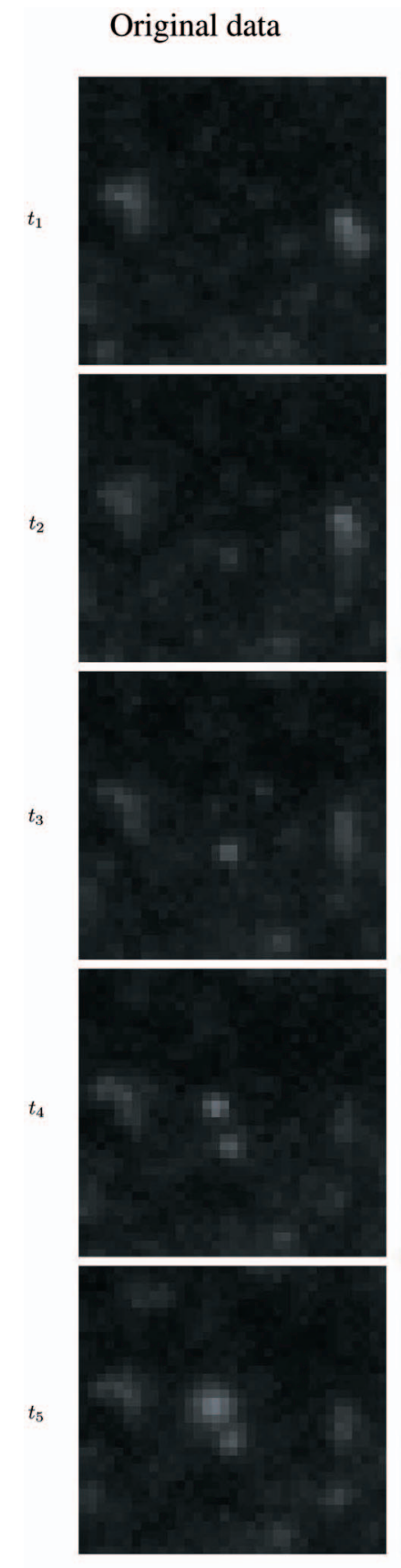

(a)
Detections
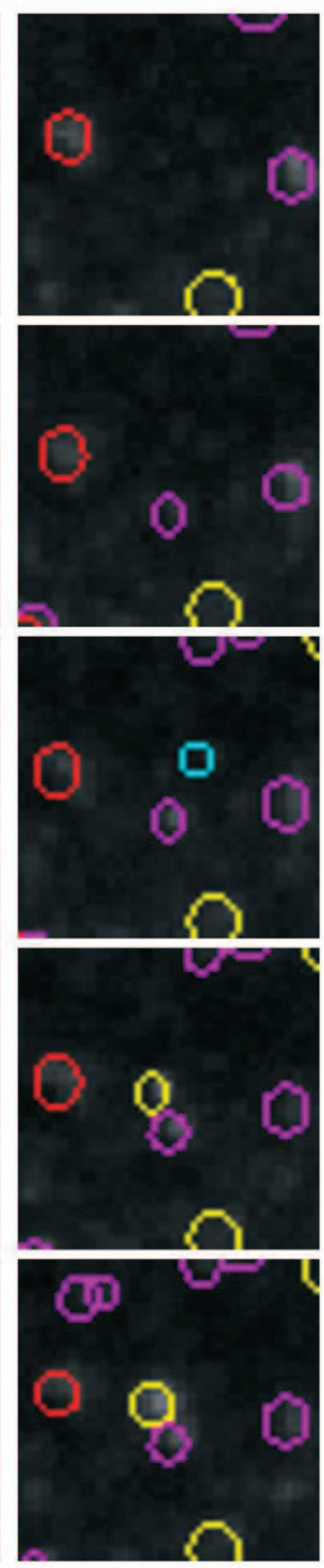

(b)
Original data
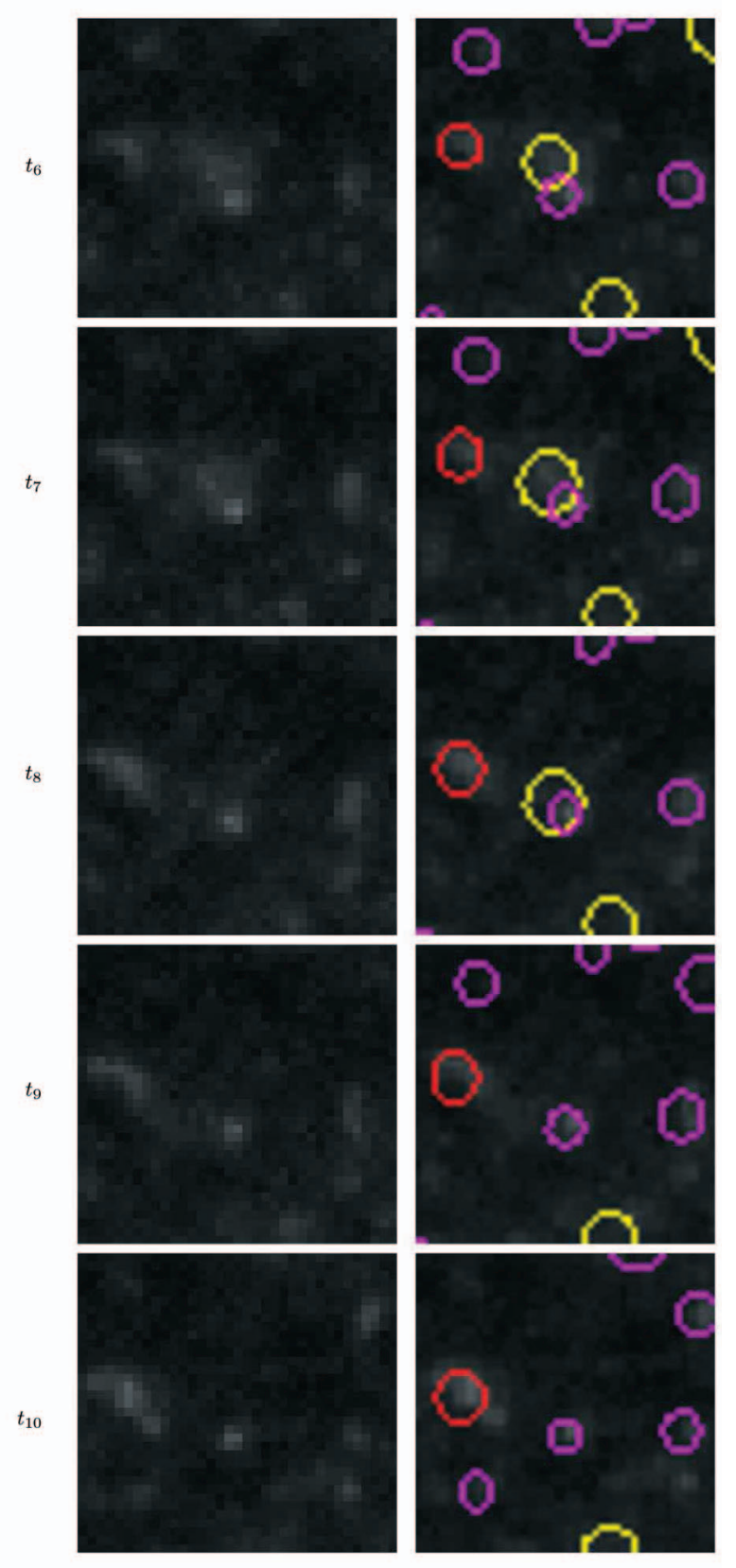

(a)

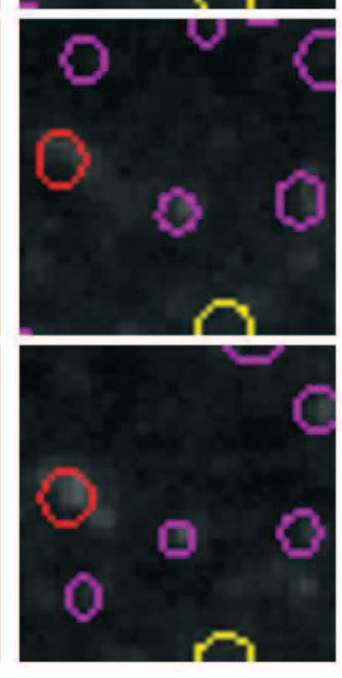

Detections
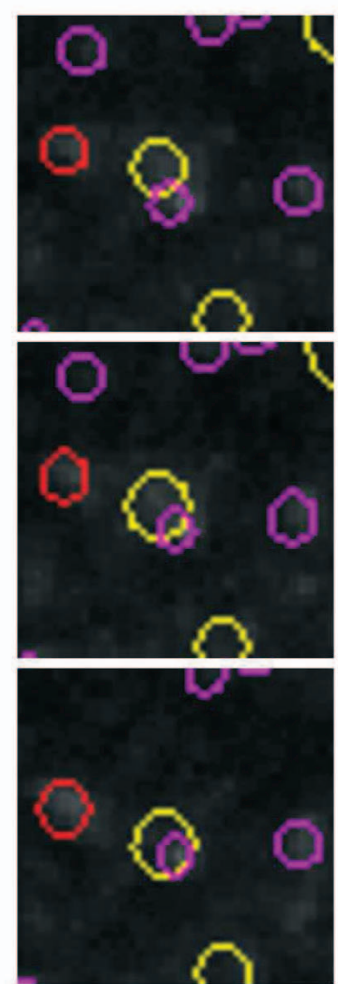

(b)

Fig. 6. Multiple vesicle tracking example [Part A]. The images show the same cell region at 10 different times. Image frames were chosen to show interesting events (frames are NOT evenly spaced in time). (a) Original data. (b) Detections made by the algorithm in the corresponding (a)-frame. Color code: [Purple] Static Vesicle. [Yellow] Fusing vesicle. Notice overlapping between a fusing vesicle and a static vesicle. [Cyan] Short-lived detection. No decision about type of dynamics can be made with a short time series. [Red] Moving vesicle (see text for more detail).

fusing vesicles, moving vesicles are completely below the surface membrane, where the excitation from the evanescent field of the TIR microscope decays exponentially as a function of depth. The low signal of late fusing vesicles is a consequence of the process of diffusion of their fluorescent tags in the cell membrane. As seen in the simulations with synthetic data (Tables 4 and 5), low values of $A$ and large values of $r$ lead to higher variance in the estimated location $\mu$ and, consequently, apparent movement, as modeled in Section 5.2.

\section{Discussion}

We have defined a statistical model for multiple objects in video image sequences and a sequence of approximations to efficiently detect events of interest. The basic objects in this case are simple "bumps," but there are already interesting issues involving object configurations and different types of motion.

In this paper, we deal with three types of vesicles: 1) fusing vesicles, 2) static vesicles, and 3) moving vesicles. A mix of these dynamics can happen, but our models do not 
TABLE 6

Size of Detection Categories with Ground Truth Labeling

\begin{tabular}{|l|c|cccc|}
\hline Cell & Frames & Fusing & Static & Moving & Total \\
\hline \hline$A$ & 14317 & 1457 & 40 & 98 & 1783 \\
$B$ & 3000 & 85 & 47 & 208 & 396 \\
\hline
\end{tabular}

cover these cases. In general, it is hard to define a set of dynamics because not much is known from the biological end. The ultimate goal of the software is to help the biologists understand the kinetics of vesicle exocytosis. Each video is intended to explore a new aspect of the problem, which implies that new types of dynamics may arise. A better understanding of the physics of the problem can help us (and the biologist) to formulate models and isolate events with specific characteristics. So far, most of the effort has been focused on detection and analysis of vesicle fusion and the events immediately before fusion.

Improvements may be possible in defining the background model. At present, a constant baseline is estimated in each region of interest. It may be possible to use earlier images to estimate a nonconstant background and improve the power of the tests. The same holds for a background model at the level of the edge features. Another direction of interest

\section{Cell A}

Cell B

(a)
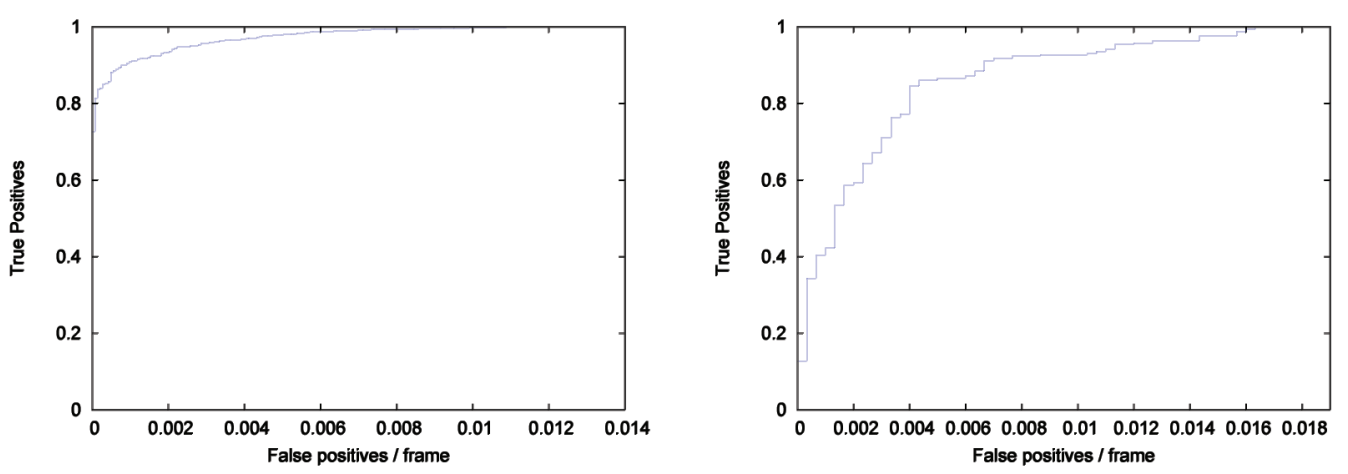

(b)
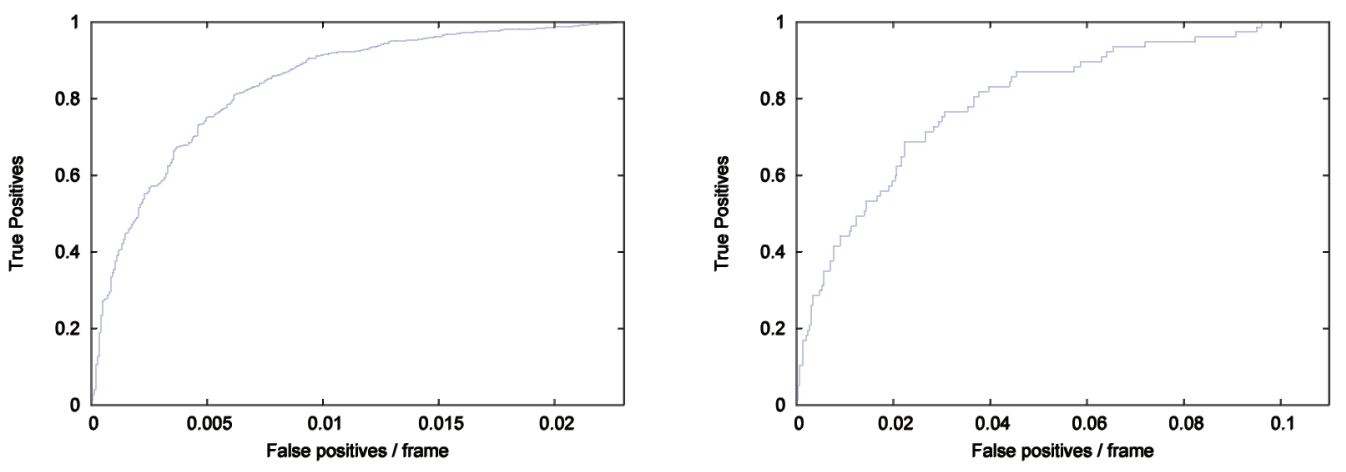

(c)
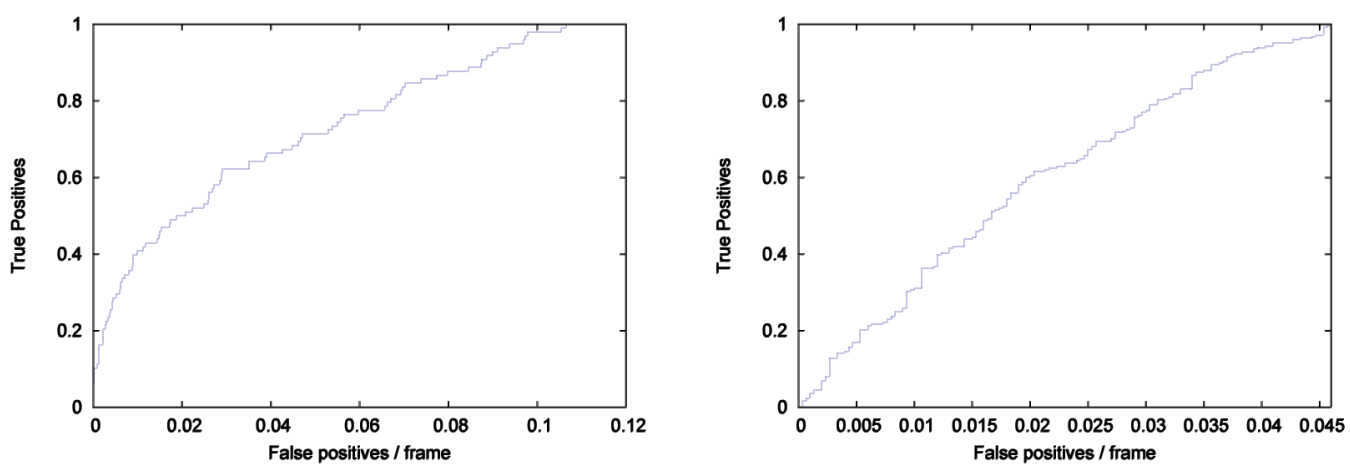

Fig. 7. ROC curves for vesicle detection and behavior classification in cells $A$ and $B$. We plot percent of true positives detected against number of false positives per frame. We labeled the ground truth on all vesicles detected by the algorithm using a very low threshold on the SNR ratio. (a) All vesicle types. (b) Fusing vesicles. (c) Moving vesicles. See Section 6.5 for more details. 
would be the online estimation of a model for a moving object using a small number of frames from motion onset.

Finally, we believe that the methodology described here can be extended to more complex objects. For example, we are currently working on tracking larger organelles with deformable shapes. The basic sequence of approximations described above will be used; however, more stages may be needed due to the more complex state variables needed to describe the object and its motion.

\section{ACKNOWLEDGMENTS}

The authors are deeply grateful to Eric Schwartz of the Department of Neurobiology, Pharmacology, and Physiology at the University of Chicago, for introducing the problem, supplying the data, encouraging the project, and providing invaluable scientific input. L. Cortés is supported in part by Burroughs Wellcome Fund Interfaces 1001774 and NSF ITR DMS-0219016. Y. Amit is supported in part by NSF ITR DMS-0219016.

\section{REFERENCES}

[1] Y. Amit, 2D Object Detection and Recognition Models, Algorithms, and Networks. MIT Press, http://mitpress.mit.edu/0262011948, 2002.

[2] Y. Amit, D. Geman, and X. Fan, "A Coarse-to-Fine Strategy for Multi-Class Shape Detection," IEEE Trans. Pattern Analysis and Machine Intelligence, vol. 26, pp. 1606-1621, 2004.

[3] Y. Amit and A. Trouvé, "Pop: Patchwork of Parts Models for Object Recognition," Int'l J. Computer Vision, 2007.

[4] E. Arias-Castro, D. Donoho, and X. Huo, "Adaptive Multiscale Detection of Filamentary Structures in a Background of Uniform Random Points," Annals of Statistics, vol. 34, pp. 326-349, 2006.

[5] E. Arias-Castro, D.L. Donoho, and X. Huo, "Near-Optimal Detection of Geometric Objects by Fast Multiscale Methods," IEEE Trans. Information Theory, vol. 51, pp. 2402-2425, 2005.

[6] S. Blackman, "Multiple Hypothesis Tracking for Multiple Target Tracking," IEEE Aerospace and Electronic Systems Magazine, vol. 19, no. 1, pp. 5-18, 2004.

[7] B. Carter, G.T. Shubeita, and S.P. Gross, "Tracking Single Particles: A User-Friendly Quantitative Evaluation," Physical Biology, vol. 2, no. 1, pp. 60-72, http://stacks.iop.org/1478-3975/2/60, 2005.

[8] M.K. Cheezum, W.F. Walker, and W.H. Guilford, "Quantitative Comparison of Algorithms for Tracking Single Fluorescent Particles," Biophysical J., vol. 81, no. 4, pp. 2378-2388, 2001.

[9] R. Deriche, "Using Canny's Criteria to Derive a Recursively Implemented Optimal Edge Detector," Int'l J. Computer Vision, vol. 1, no. 2, pp. 167-187, May 1987.

[10] F. Fleuret and D. Geman, "Coarse-to-Fine Face Detection," Int'l J. Computer Vision, vol. 41, nos. 1/2, pp. 85-107, 2001.

[11] T. Fortmann, Y. Bar Shalom, and M. Scheffe, "Sonar Tracking of Multiple Targets Using Joint Probabilistic Data Association," IEEE J. Oceanic Eng., vol. 8, no. 3, pp. 173-184, July 1983.

[12] M. Isard and A. Blake, "Condensation-Conditional Density Propagation for Visual Tracking," Int'l J. Computer Vision, vol. 29, no. 1, pp. 5-28, 1998.

[13] M. Isard and J. MacCormick, "Bramble: A Bayesian Multiple-Blob Tracker," Proc. Eighth IEEE Int'l Conf. Computer Vision, pp. 34-41, 2001.

[14] L. Johnston and V. Krishnamurthy, "Performance Analysis of a Dynamic Programming Track Before Detect Algorithm," IEEE Trans. Aerospace and Electronic Systems, vol. 38, no. 1, pp. 228-242, Jan. 2002.

[15] C.H. Li, L. Bai, D.D. Li, S. Xia, and T. Xu, "Dynamic Tracking and Mobility Analysis of Single Glut4 Storage Vesicle in Live 3t3-11 Cells," Cell Research, vol. 14, no. 6, pp. 480-486, 2004.

[16] E. Meijering, I. Smal, and G. Danuser, "Tracking in Molecular Bioimaging," IEEE Signal Processing Magazine, vol. 23, no. 3, pp. 46-53, http://ieeexplore.ieee.org/xpls/abs_all.jsp?arnumber $=1628877,2006$.
[17] C. Rasmussen and G.D. Hager, "Probabilistic Data Association Methods for Tracking Complex Visual Objects," IEEE Trans. Pattern Analysis and Machine Intelligence, vol. 23, no. 6, pp. 560-576, June 2001.

[18] D. Reid, "An Algorithm for Tracking Multiple Targets," IEEE Trans. Automatic Control, vol. 24, no. 6, pp. 843-854, Dec. 1979.

[19] J.A. Rice, Mathematical Statistics and Data Analysis. Duxbury Press, 1994.

[20] A. Santos and I.T. Young, "Model-Based Resolution: Applying the Theory in Quantitative Microscopy," Applied Optics, vol. 39, pp. 2948-2958, June 2000.

[21] I. Sbalzarini and P. Koumoutsakos, "Feature Point Tracking and Trajectory Analysis for Video Imaging in Cell Biology," J. Structural Biology, vol. 151, no. 2, pp. 182-195, 2005.

[22] J. Steyer and W. Almers, "A Real-Time View of Life within $100 \mathrm{~nm}$ of the Plasma Membrane," Nature Rev. Molecular Cell Biology, vol. 2, pp. 268-275, 2001.

[23] T. Südhof, "The Synaptic Vesicle Cycle," Ann. Rev. Neuroscience, vol. 27, pp. 509-547, 2004.

[24] D. Thomann, J. Dorn, P. Sorger, and G. Danuser, "Automatic Fluorescent Tag Localization. II: Improvement in Super-Resolution by Relative Tracking," J. Microscopy, vol. 211, pp. 230-248, Sept. 2003.

[25] D. Thomann, D. Rines, P. Sorger, and G. Danuser, "Automatic Fluorescent Tag Detection in 3D with Super-Resolution: Application to the Analysis of Chromosome Movement," J. Microscopy, vol. 208, pp. 49-64, Oct. 2003.

[26] S. Tonissen and Y. Bar-Shalom, "Maximum Likelihood TrackBefore-Detect with Fluctuating Target Amplitude," IEEE Trans. Aerospace and Electronic Systems, vol. 34, no. 3, pp. 796-809, July 1998.

[27] P. Viola and M. Jones, "Robust Real-Time Face Detection," Proc. Eighth IEEE Int'l Conf. Computer Vision, vol. 2, pp. 747-747, 2001.

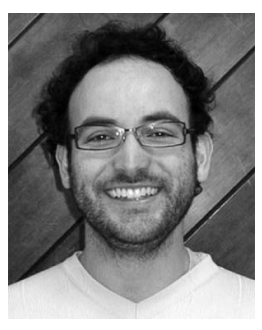

Leandro Cortés received the degree in computer engineering from the National University of Colombia in 1998 and the MSc degree in computer science from the University of Chicago in 2004, where he is currently working toward the PhD degree in computer science. His research interests include computer vision, cellular microscopy, image processing, and machine learning. He is a student member of the IEEE.

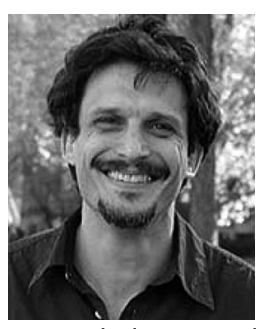

Yali Amit received the PhD degree in mathematics from the Weizmann Institute, Israel in 1988. He spent three years as a visiting assistant professor in the Division of Applied Math at Brown University, where he started working on image analysis. In 1991, he joined the Department of Statistics at the University of Chicago. In 2000, he was appointed a full professor with a joint appointment in statistics and computer science. In recent years, his research interests include object detection and recognition, speech recognition, machine learning, and computational models for the biological visual system. In 2002, he published the book 2D Object Detection and Recognition: Models, Algorithms and Networks (MIT Press). He is a member of the IEEE.

$\triangleright$ For more information on this or any other computing topic, please visit our Digital Library at www.computer.org/publications/dlib. 